\title{
Primary pure non-gestational choriocarcinoma of ovary: a rare case report
}

\section{Gireesha Rawal, Charanjeet Ahluwalia*, Amit Kumar Yadav, Indrani Dhawan}

\author{
Department of Pathology, Vardhman Mahavir Medical College and Safdarjung Hospital, New Delhi, India
}

Received: 10 July 2017

Accepted: 05 August 2017

\section{*Correspondence:}

Dr. Charanjeet Ahluwalia,

E-mail: charanjeet.ahluwalia@ rediffmail.com

Copyright: (C) the author(s), publisher and licensee Medip Academy. This is an open-access article distributed under the terms of the Creative Commons Attribution Non-Commercial License, which permits unrestricted non-commercial use, distribution, and reproduction in any medium, provided the original work is properly cited.

\begin{abstract}
Germ cell malignancies represent $15 \%$ of ovarian cancers. Choriocarcinoma is a malignant tumour with trophoblastic differentiation. It may be gestational or non-gestational and may be a primary tumour or metastatic from other organs. Pure non-gestational choriocarcinoma is an extremely rare neoplasm, with reports of only a few cases. This study reports a rare case of non-gestational pure choriocarcinoma in a postmenarcheal young female and describes details of the tumour, including the clinicopathological findings. A young female presented with sudden onset anxiety, abdominal distention, severe abdominal pain, and severe pallor. Clinically, she was diagnosed as a case of ruptured ectopic pregnancy, and was taken up for emergency laparotomy. A right ovarian mass of $5 \mathrm{X} 7 \mathrm{~cm}$ was seen, which was ruptured and was bleeding profusely. Right salpingo-oophorectomy was done, and it was diagnosed histopathologically as primary pure non-gestational choriocarcinoma of ovary. This case report describes the histopathological and immunohistochemical findings of this rare entity, as well as the various methods to differentiate between cases of non-gestational and gestational choriocarcinoma of the ovary. This is important as non-gestational choriocarcinoma has a poorer prognosis in comparison with gestational. Also, the treatment regimen for the two differs.
\end{abstract}

Keywords: Choriocarcinoma, Non-gestational, Pure

\section{INTRODUCTION}

Germ cell malignancies represent $15 \%$ of ovarian cancers in Asian and African-American nations. ${ }^{1}$ Choriocarcinoma is a malignant tumour with trophoblastic differentiation. It may be gestational or nongestational choriocarcinoma and may be a primary tumour or metastatic from other organs.

Non-gestational choriocarcinoma is usually a component of a mixed germ cell tumour., ${ }^{2,3}$ Pure non-gestational choriocarcinoma of the ovary is an extremely rare neoplasm, with only a few reported cases in literature. ${ }^{4,5}$ In general, majority of the non-gestational type have been clinically diagnosed in patients who were sexually immature, unable to conceive or had never had sexual intercourse. Pure choriocarcinoma has been defined as a tumour that does not include other germ cell tumour elements. ${ }^{2}$

It is important to differentiate between cases of nongestational or gestational choriocarcinoma of the ovary, as non-gestational choriocarcinoma has a poorer prognosis compared to gestational. Also, the treatment regimen for the two differs. In the absence of clinical information, it is exceedingly difficult to differentiate the two morphologically. This study reports a rare case of primary pure non-gestational choriocarcinoma of ovary in a postmenarcheal young female and describes details of the tumour, including the clinicopathological findings. 


\section{CASE REPORT}

A 25 years old female, $\mathrm{P}_{3} \mathrm{~L}_{3}$, presented to our hospital with sudden onset anxiety, abdominal distention and severe abdominal pain, along with severe pallor. Her LMP (Last Menstrual Period) was 1 week prior to the date of presentation. She had a history of a full term normal vaginal delivery 5 months back. Following that, she had normal regular cycles. On per-abdominal examination, abdomen was distended with fluid. On pervaginal examination, os was closed and bilateral fornices were full. Clinically, she was diagnosed as a case of ruptured ectopic pregnancy, and was taken up for emergency laparotomy. Per-operatively, 2 litres of haemoperitoneum was found and drained. A right ovarian mass of $5 \times 7 \mathrm{~cm}$ was seen, which was ruptured and was bleeding profusely.

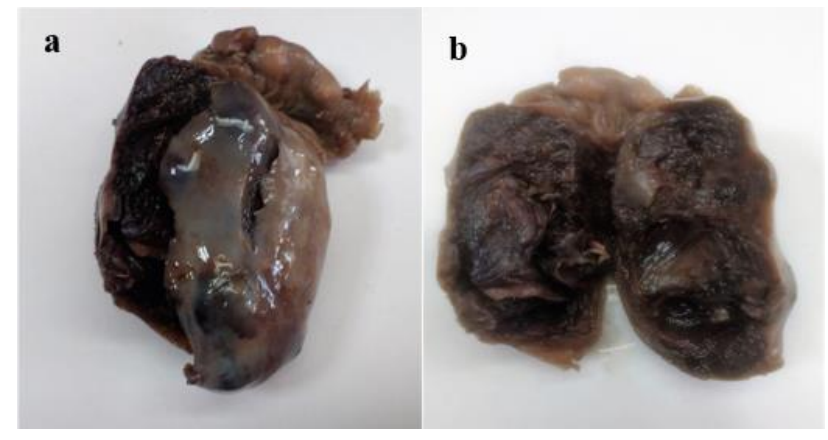

Figure 1: a) Gross showing enlarged ovary, congested external surface and attached fallopian tube. b) Cut section of ovary showing a grey white tumour with areas of haemorrhage and necrosis.

Right salpingo-oophorectomy was done, and the specimen was sent for histopathology. Grossly the ovary was enlarged, measuring $5 \times 3 \times 2 \mathrm{~cm}$. External surface was congested, and a rupture site was identified $0.5 \mathrm{~cm}$ in diameter. Attached fallopian tube was $4 \mathrm{~cm}$ in length (Figure 1a). Cut section of ovary showed a grey white tumour with areas of haemorrhage and necrosis (Figure $1 b)$.
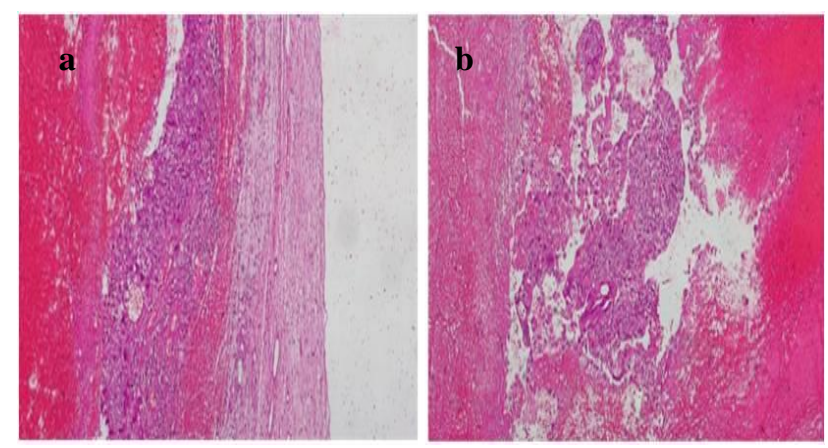

Figure 2(a): Microscopy sections showing normal ovarian stroma along with tumour (X10) (b): Tumour is composed of sheets of cytotrophoblastic and syncytiotrophoblastic cells, along with extensive areas of haemorrhage and necrosis (X10).
The specimen was grossed and processed. On microscopy, sections showed a tumour composed of sheets of cytotrophoblastic and syncytiotrophoblastic cells, along with extensive areas of haemorrhage and necrosis(Figure $2 \mathrm{a}$ and $\mathrm{b}$ ). The trophoblastic cells were highly pleomorphic with irregular hyperchromatic nuclei (Figure 3a). Even on extensive sampling, no other germ cell element could be found. Attached fallopian tube was unremarkable. Immunohistochemistry for beta-HCG, CD30, PLAP and AFP was performed. The tumour cells showed strong cytoplasmic positivity for beta HCG (Figure $3 \mathrm{~b}$ ), while the rest of the markers were negative, thus confirming our diagnosis.

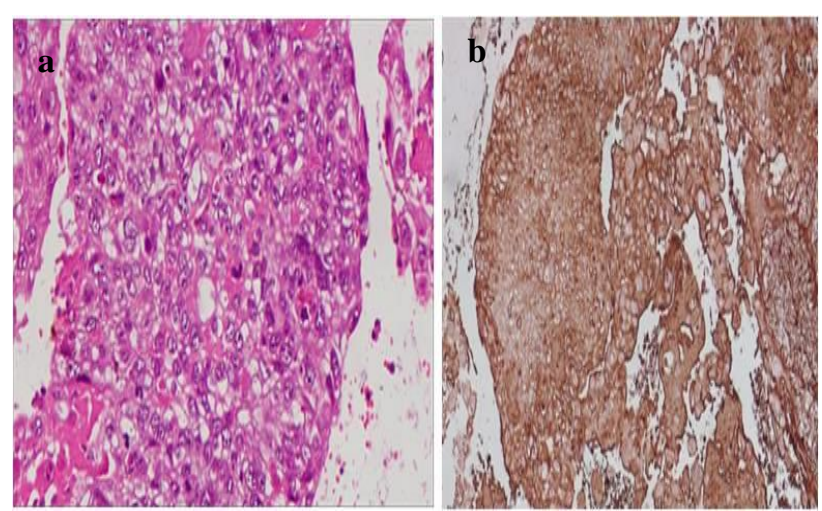

Figure 3 (a): Trophoblastic cells are highly pleomorphic with irregular hyperchromatic nuclei (X40) (b): IHC showing positivity for beta HCG (X10).

The beta- HCG level of the patient was very high $(80,000$ IU). An abdominal USG revealed no evidence of uterine pregnancy, ectopic pregnancy, or uterine choriocarcinoma. Hence, a final diagnosis of primary pure non-gestational choriocarcinoma of ovary was given.

\section{DISCUSSION}

Two forms of ovarian choriocarcinoma have been reported, gestational and non-gestational. Gestational choriocarcinoma of the ovary results from metastasis of a uterine trophoblastic disease, or, rarely, following an ovarian pregnancy. The non-gestational type is described as a primary germ cell neoplasm differentiating along the extraembryonic chorionic tissue. The majority of nongestational tumours occur in combination with other germ cell neoplasms.

Pure non-gestational choriocarcinoma of the ovary is defined as a tumour that occurs in the absence of any other germ cell tumours. It is extremely rare, ${ }^{2,3}$ and very few cases of this type of choriocarcinoma have been previously described. ${ }^{4,5}$ It most commonly occurs in young females. In the present case of a 25 years old female, no additional components of any germ cell tumour were found in association with the 
choriocarcinoma. Moreover, the histological examination revealed no similar neoplasmss in other organs.

It is important to differentiate between cases of nongestational and gestational choriocarcinoma of the ovary, as non-gestational choriocarcinoma has a poorer prognosis in comparison with gestational. ${ }^{6}$ Pure nongestational ovarian choriocarcinomas are extremely rare and highly malignant tumours which frequently metastasize through the lymphatics with intra-abdominal spread. ${ }^{7,8}$ Also, the treatment regimen for the two differs.

The gestational variants are extremely sensitive to chemotherapy with cure rate approaching 98\%. ${ }^{9}$ Non gestational choriocarcinomas are resistant to single-agent chemotherapy and have a worse prognosis as compared to gestational choriocarcinomas. ${ }^{10}$ Morphologically, the presence of a well developed corpus luteum of pregnancy adjacent to the tumour may be indicative of a gestational origin. $^{7}$ This was not seen in our case.

Other way of differentiating gestational from nongestational choriocarcinoma is by using markers. Several previous studies have investigated the high expression levels of $\beta 2$-microglobulin (BMG) in choriocarcinoma. Tanaka et al ${ }^{11}$ reported a lack of effective messenger RNA for BMG in gestational human choriocarcinoma cell lines, as well as the presence of messenger RNA for $\mathrm{BMG}$ in non-gestational choriocarcinoma.

Studies by Kato et al $^{12}$ and Norman et al ${ }^{13}$ indicated that BMG may be clinically used as a serum marker for nongestational choriocarcinoma. In another study, BMG was detected in tumour cells of non-gestational choriocarcinoma using immunohistochemistry. ${ }^{14}$

A search for paternal DNA in tumour allows a definite distinction between gestational and non-gestational types. Tumours with gestational origin have paternal genomic structure while nongestational tumours have genomes of only maternal origin without any alleles from paternal origin. $^{15}$

\section{CONCLUSION}

In conclusion, we present a case of primary pure nongestational choriocarcinoma of ovary in a 25 years old female, wherein there was absence of any other germ cell component, and there was no evidence of uterine pregnancy, ectopic pregnancy, or uterine choriocarcinoma.

It is important to differentiate between cases of nongestational or gestational choriocarcinoma of the ovary, as cases of non-gestational choriocarcinoma have a poorer prognosis compared with gestational cases. Also, the treatment regimen for the two differs.

More pathological and biological techniques for the differentiation of gestational and non-gestational tumours are required for the efficient treatment of choriocarcinoma in the future.

Funding: No funding sources

Conflict of interest: None declared

Ethical approval: Note required

\section{REFERENCES}

1. Corakçi A, Ozeren S, Ozkan S, Gürbüz Y, Ustün H, Yücesoy I. Pure nongestational choriocarcinoma of ovary. Arch Gynecol Obstet. 2005;271(2):176-7.

2. Russell P, Farnsworth A, editors. Surgical Pathology of the Ovaries. $2^{\text {nd }}$ ed. Churchill Livingstone; Edinburgh: Non-gestational choriocarcinomas; 1997:263-4.

3. Scully RE, Young RH, Clement PB, editors. Tumors of the Ovary, Maldeveloped Gonads, Fallopian Tube, and Broad Ligament. Atlas of Tumor Pathology. 3rd series. Fascicle. Vol. 23. Armed Forces Institute of Pathology; Washington DC: Choriocarcinoma; 1998:258-60.

4. Goswami D, Sharma K, Zutshi V, Tempe A, Nigam S. Nongestational pure ovarian choriocarcinoma with contralateral teratoma. Gynecol Oncol. 2001;80:2626.

5. Inaba H, Kawasaki H, Hamazaki M, Okugawa T, Uchida K, Honzumi M, et al. A case of metastatic ovarian non-gestational choriocarcinoma: successful treatment with conservative type surgery and myeloablative chemotherapy. Pediatr Int. 2000;42:383-5.

6. Scully RE. Hartmann WH, editor. Tumors of the ovary and mal-developed gonads. Atlas of tumor pathology. 1979:243-5.

7. Russell P, Farnsworth A. Surgical pathology of the ovaries. $2^{\text {nd }}$ sub ed. Philadelphia: W.B. Saunders Company; 1997:785-7.

8. Gerbie MV, Brewer JI, Tamimi H. Primary choriocarcinoma of the ovary. Obstet Gynecol. 1975;46(6):720-3.

9. Lorigan PC, Colman RE, Hancock BW. The treatments of persistent trophoblastic disease using the Sheffield modification of chaing cross risk score. Proc Annu Meeting Am Soc Clin Oncol. 1994;13:257.

10. Rao KVLN, Konar S, Gangadharan J, Vikas V, Sampath S. A pure non-gestational ovarian choriocarcinoma with delayed solitary brain metastases: Case report and review of the literature. J Neurosci Rural Pract. 2015;6(4):578-81.

11. Tanaka K, Nabeshima Y, Takahashi H, Takeuchi S, Nabeshima Y, Ogata K. Lack of effective messenger RNA for beta 2-microglobulin in a gestational human choriocarcinoma cell line (GCH-1). Cancer Res. 1981;41(9 Pt 1):3639-41.

12. Kato M, Ohashi K, Saji F, Wakimoto A, Tanizawa O. Expression of HLA class I and beta 2microglobulin on human choriocarcinoma cell lines: 
induction of HLA class I by interferon-gamma. Placenta. 1991;12(3):217-26.

13. Norman RJ, Jialal I, Joubert SM, Green-Thompson RW. Beta-2-microglobulin in trophoblastic disease. S Afr Med J. 1983;64(3):90-2.

14. Hayashi S, Abe Y, Tomita S, Nakanishi Y, Miwa S, Nakajima $T$, et al. Primary non-gestational pure choriocarcinoma arising in the ovary: A case report and literature review. Oncology Letters. 2015;9(5):2109-2111.
15. Koo HL, Choi J, Kim KR, Kim JH. Pure nongestational choriocarcinoma of the ovary diagnosed by DNA polymorphism analysis. Pathol Int. 2006;56(10):613-6.

Cite this article as: Rawal G, Ahluwali C, Yadav AK, Dhawan I. Primary pure non-gestational choriocarcinoma of ovary: a rare case report. Int J Reprod Contracept Obstet Gynecol 2017;6:4166-9. 